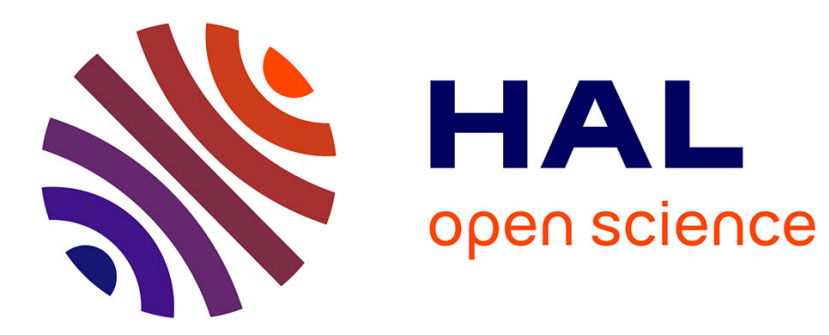

\title{
DISORDERING TRANSITIONS OF METAL SURFACES
}

G. Held, J. Jordan-Sweet, P. Horn, A. Mak, R. Birgeneau

\section{To cite this version:}

G. Held, J. Jordan-Sweet, P. Horn, A. Mak, R. Birgeneau. DISORDERING TRANSITIONS

OF METAL SURFACES. Journal de Physique Colloques, 1989, 50 (C7), pp.C7-245-C7-255. 10.1051/jphyscol:1989726 . jpa-00229893

\section{HAL Id: jpa-00229893 https://hal.science/jpa-00229893}

Submitted on 1 Jan 1989

HAL is a multi-disciplinary open access archive for the deposit and dissemination of scientific research documents, whether they are published or not. The documents may come from teaching and research institutions in France or abroad, or from public or private research centers.
L'archive ouverte pluridisciplinaire HAL, est destinée au dépôt et à la diffusion de documents scientifiques de niveau recherche, publiés ou non, émanant des établissements d'enseignement et de recherche français ou étrangers, des laboratoires publics ou privés. 
DISORDERING TRANSITIONS OF METAL SURFACES

\author{
G.A. HELD, J.L. JORDAN-SWEET, P.M. HORN, A. MAK ${ }^{*}$ and R.J. BIRGENEAU* \\ IBM Research Division, Thomas J. Watson Research Center, Yorktown \\ Heights, NY 10598-0218, U.S.A. \\ *Department of Physics, Massachusetts Intitute of Technology, \\ Cambridge, MA 02139, U.S.A.
}

\begin{abstract}
Résumé - Nous présentons ici une revue de résultats récents obtenus par diffusion de rayons $\mathrm{X}$ provenant d'une source synchrotron. Nous avons étudié la rugosité thermique de $\mathrm{Ag}$ (110), ainsi que la rugosité et la déconstruction de la surstructure $1 \times 3$ de $\mathrm{Au}$ (110). Les expériences sur $\mathrm{Ag}$ (110) mettent en évidence une transition rugueuse à $T_{R}=450^{\circ} \mathrm{C}$. Au dessus de $T_{R}$, la phase rugueuse est caractérisée par des fluctuations de hauteur à divergence logarithmique. Pour la face (110) de l'or, on trouve qu'à $\mathrm{T}_{\mathrm{C}}=485^{\circ} \mathrm{C}$ la surstructure $1 \times 3$ subit une transition réversible vers une structure incommensurable. Les mesures suggèrent que la surface devient à la fois rugueuse et se deconstruit au cours de la transition commensurable-incommensurable.
\end{abstract}

\begin{abstract}
We review the results of recent synchrotron x-ray scattering experiments which study the thermal roughening of $\mathrm{Ag}(110)$ as well as the roughening and deconstruction of the $\mathrm{Au}(110)$ $1 \times 3$ reconstruction. The $\mathrm{Ag}(110)$ experiments provide direct evidence that the silver surface undergoes a roughening transition at $T_{R}=450^{\circ} \mathrm{C}$. Above $T_{R}$, the rough phase is characterized by logarithmically divergent height fluctuations. For $\mathrm{Au}(110)$, it is found that at $\mathrm{T}_{c}=485^{\circ} \mathrm{C}$ the $1 \times 3$ surface undergoes a reversible transition to a structure incommensurate with the bulk crystalline lattice. The data suggest that during this commensurate-incommensurate transition the surface both roughens and deconstructs.
\end{abstract}

\title{
INTRODUCTION
}

Recent experiments have demonstrated that $x$-ray scattering is an effective means of studying surface phase transitions. Specifically, the lineshapes of bulk-forbidden surface peaks can provide direct information about the surface height-height (or step-step) correlation functions and are thus a sensitive probe of the surface roughening transition [1]. This transition is characterized by a proliferation of atomic steps which results in logarithmically divergent height fluctuations of the crystalline surface $[2,3]$.

We have carried out glancing angle synchrotron $x$-ray scattering experiments as a means of studying the roughening of the $\mathrm{Ag}(110)$ surface as well as the $\mathrm{Au}(110) 1 \times 3$ to $1 \times 1$ deconstruction transition. For the

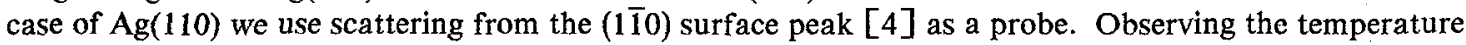
dependence of this bulk forbidden peak, we find that the surface undergoes a roughening transition at $T_{R}=450 \pm 25^{\circ} \mathrm{C}$. Above $T_{R}$, the rough phase is characterized by logarithmically divergent height fluctuations. In the case of the $\mathrm{Au}(110) 1 \times 3$ reconstructed surface, superlattice peaks as well as integral order bulk-forbidden surface peaks are present. Observing the temperature dependence of these peaks, we find that at $\mathrm{T}_{\mathrm{c}}=485^{\circ} \mathrm{C}$ the $\mathrm{Au}(110) 1 \times 3$ surface becomes incommensurate with the bulk crystalline lattice. During the commensurate-incommensurate transition, the surface both roughens and deconstructs. Preliminary reports of these results have been published elsewhere $[1,5]$. 


\section{EXPERIMENTAL PROCEDURES}

In order to carry out these experiments, we constructed a UHV chamber equipped with the instrumentation needed to prepare a clean surface, and yet compact enough to fit into a Huber 6-circle diffractometer, thereby allowing the precise sample orientation essential for high resolution $\mathrm{X}$-ray scattering measurements. Our chamber was constructed from a $6^{\prime \prime}$ diameter by $12^{\prime \prime}$ long stainless steel cylindrical tube. A turbomolecular pump and a 4-grid rear-view LEED system were mounted on opposite ends, while the sample holder, ion sputtering gun, ionization gauge, and gas inlet value were mounted along the cylindrical surface. X-rays entered and exited the chamber via two $120^{\circ}$ Be windows which were welded opposite one another onto the cylindrical surface of the chamber. The base pressure of this system was $5 \times 10^{-10}$ Torr.

The $\mathrm{Ag}(110)$ surface was mechanically polished and etched; the $\mathrm{Au}(110)$ surface was electropolished. Both samples were susequently cleaned by cycles of Ar-ion sputtering and annealing until a well ordered surface was obtained. The surface miscuts of the $\mathrm{Ag}(110)$ and $\mathrm{Au}(110)$ surfaces were measured to be $\$ 0.3^{\circ}$ and $0.2^{\circ}$, respectively.

The scattering measurements were taken in glancing angle geometry with an incident beam of $8.05 \mathrm{keV}$ photons focussed on the samples. The Ag scattering measurements were taken at the IBM/MIT beamline $\mathrm{X}-20 \mathrm{~A}$ at the National Synchrotron Light Source and the Au data were taken at the eight-pole wiggler beamline VII-2 at SSRL (SLAC). For the Ag experiments the longitudinal (radial) resolution of the spectrometer was set by slits at $0.0044 \AA^{-1}$, less than one-half the radial width of the low temperature $\mathrm{Ag}$ (1T0) surface peak. For the Au experiments the resolution in the $2-\theta$ direction of the spectrometer was set by slits at $0.014 \AA^{-1}$ HWHM. The transverse resolution within the scattering plane was $2 \times 10^{-4} \AA^{-1}$ HWHM.

\section{SILVER (110)}

The scattering from a smooth surface is a series of Bragg rods with delta function cross sections. There exist specific points along these rods at which scattering from alternate planes of atoms exactly cancel. These points (known as bulk forbidden or anti-Bragg points) are sensitive to surface steps. When the surface becomes logarithmically rough, the scattering from these points evolves from a delta function into a power law lineshape with an exponent $\eta$. This exponent is a direct measure of the rate of the logarithmic divergence of the mean-square height fluctuations of the surface, and, hence, of the surface roughness. We find that at $450 \pm 25^{\circ} \mathrm{C}$, well below the bulk melting point, the (110) surface peak of the $\mathrm{Ag}$ (110) surface undergoes an evolution from delta-function to power law lineshape, providing direct confirmation of the existence of a logarithmically rough surface.

As shown in Fig. 1, between $100^{\circ}$ and $550^{\circ} \mathrm{C}$, the $\mathrm{Ag}(1 \mathrm{10})$ surface peak udergoes radical changes in intensity and lineshape. At $100^{\circ} \mathrm{C}$ the scattering is sharply peaked. The lineshape changes only slightly until approximately $375^{\circ} \mathrm{C}$, at which point it begins to develop substantial tails, as well as an appreciable loss in intensity. By $450^{\circ} \mathrm{C}$ the scattering is comprised entirely of power law tails, as will be discussed below. The transition shown here is reversible with temperature, indicating that it is indeed an equilibrium phase transition and not a non-equilibrium micro-faceting process. Between $100^{\circ}$ and $500^{\circ} \mathrm{C}$, the integrated intensity of the bulk $\mathrm{Ag}(1 \overline{1} 1)$ peak decreases only by $20 \%$ (the expected Debye-Waller factor) and undergoes no change in lineshape, demonstrating that the observed changes in the bulk forbidden (110) peak are due exclusively to changes in the surface morphology.

Theoretical studies of thermal roughening predict specific lineshapes for atomic scattering from surfaces both at $T<<T_{R}$ and $T>T_{R}$ [3]. Following Ref. 4, it is straight forward to show that these results are applicable to $x$-ray scattering from bulk-forbidden surface peaks as well. For- $T<<T_{R}$ the surface 
has well defined long range order (LRO) and the surface peaks should be delta functions. For $T>T_{R}$ the logarithmic divergence of the height fluctuations results in an algebraic decay of the LRO. That is [6],

$$
<\rho_{\mathbf{G}}(\mathbf{r}) \rho_{-\mathbf{G}}(0)>\sim\left(1+\mathrm{x}^{2} / \xi_{\mathrm{x}}^{2}+\mathrm{y}^{2} / \xi_{\mathrm{y}}^{2}\right)^{-\eta_{\mathbf{G}} / 2}
$$

where $\rho_{\mathbf{G}}$ is the Fourier component of the mass density with reciprocal lattice vector $\mathbf{G}$ (in our case $(1 \overline{10})$ ), $\xi_{\mathrm{x}}$ and $\xi_{\mathrm{y}}$ are scaling lengths in the transverse and radial directions respectively, and $\eta$ (dropping the subscript) increases with temperature above $T_{R}$. The value of $\eta$ at the transition temperature $\eta_{R}$ is predicted by several exactly solvable models [3] to equal unity at the (110) anti-Bragg position. Algebraic decay of $L R O$ results in a scattering cross section $S(Q)$ proportional to $\left|\xi_{y} q_{y}\right|^{-2+\eta}$ and $\left|\xi_{x} q_{x}\right|^{-2+\eta}$ in the radial and transverse directions, respectively, where $\mathbf{q} \equiv \mathbf{Q}-\mathbf{G}$ is the distance in reciprocal space from the $(1 \overline{1} 0)$ wavevector and $1 / \mathrm{q}>\xi_{\mathrm{x}}, \xi_{\mathrm{y}}$. For $\mathrm{T} \lesssim \mathrm{T}_{\mathrm{R}}$ one expects the scattering to be a superposition of a delta function (Bragg scattering) and diffuse tails from the local steps [3]. As $T_{R}$ is approached from below, the intensity of the Bragg component of the scattering goes to zero.

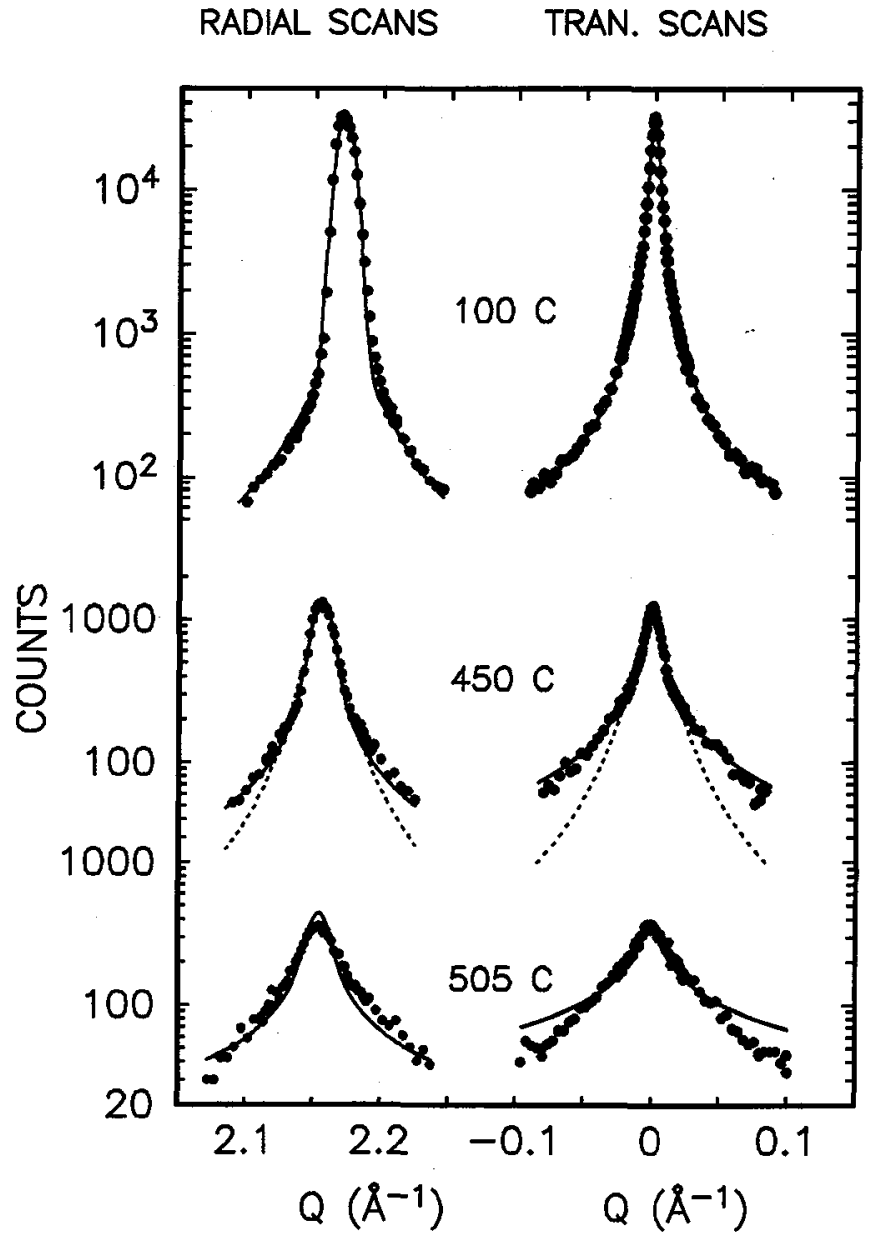

Fig. 1 - Radial and transverse scans through the Ag (110) surface peak at temperatures below, near, and above the roughening transition. The solid lines through the data are best fits to the power law lineshapes discussed in the text. The exponent $\eta$ is equal to $0,0.77$, and 1.15 for the fits at 100,450 , and $505^{\circ} \mathrm{C}$, respectively. The dashed lines through the $450^{\circ} \mathrm{C}$ data are best fit Lorentzians. Background scattering has been subtracted from all of the data shown. Count rate shown is per 5 seconds. 
For any physical system, both delta-function and power law lineshapes are modified by the finite size of the coherent surface domains, as well as by the finite resolution of the $\mathrm{x}$-ray spectrometer. At $100^{\circ} \mathrm{C}$ our radial profile consists of a sharp center portion and broad, low intensity tails. Empirically, this is well described as the sum of a Gaussian and a Lorentzian function with widths of $0.0054 \AA^{-1}$ and 0.0238 $\AA^{-1}$, respectively (prior to convolution with the slit resolution function), and relative peak intensities of 56:1. Our mean domain size is thus $\cong 600 \AA$. Correspondingly, the transverse scan, which at this temperature is dominated by the surface mosaic, is well described as the sum of two Lorentzians. These fits are shown as solid lines for the $100^{\circ} \mathrm{C}$ data in Fig. 1. The high quality of our sample clearly allows one to obtain lineshape information in both the radial and the transverse directions; at $100^{\circ} \mathrm{C}$ our signal-tobackground ratio is $\geq 1500: 1$.

With increasing temperature, the peak-to-tail ratio of the $(1 \overline{1} 0)$ lineshape steadily decreases. To quantify this evolution, we fit the data as a superposition of the low temperature lineshape multiplied by a temperature dependent scale factor, together with additional, thermally induced tails. Thus, we are able to determine the 2-dimensional integrated intensity of the entire (1i10) peak, as well as that of its components, as a function of temperature. In Fig. 2(a) we plot the intensity of the entire (110) peak as closed circles, and show the Bragg intensity (i.e., the scattering not included in the temperature dependent tails) as open circles. Theoretically, it is expected that the Bragg intensity will fall to zero at $T_{R}$, and we find that this happens at approximately $450^{\circ} \mathrm{C}$. The total intensity falls off much more slowly.

For $\mathrm{T}>\mathrm{T}_{\mathbf{R}}$, the surface peak has no Bragg component; it consists entirely of a power law lineshape. To fit a power law to the data, it is first necessary to take into account the finite size effects of the surface. For a Gaussian distribution of domains, Dutta and Sinha (DS) [7] have shown that the scattering is accurately described by a Kummer function,

$$
\mathrm{S}(\mathbf{Q})=\mathrm{A}(\mathrm{L} / \xi)^{2-\eta} \Phi\left(1-\eta / 2 ; 1 ;-\mathrm{q}^{2} \mathrm{~L}^{2} / 4 \pi\right)
$$

where $\mathrm{L}$ is the mean domain size and $\mathrm{A}$ is independent of $\mathrm{q}$. This function has a Gaussian center portion of HWHM $\pi / L$, tails proportional to $|1 / q \xi|^{2-\eta}$ for $q>>(1 / L)$, and reduces to a Gaussian for $\eta=0$. For a Lorentzian distribution of domains, the corresponding function has the form

$$
\mathrm{S}(\mathbf{Q})=\mathrm{B}(\mathrm{L} / \xi)^{2-\eta} \mathrm{F}\left(1-\eta / 2,1-\eta / 2 ; 1 ;-\mathrm{q}^{2} \mathrm{~L}^{2} / \pi^{2}\right)
$$

where $\mathrm{F}$ is a hypergeometric function, $\pi / \mathrm{L}$ is the HWHM of the Lorentzian and B is independent of $\mathrm{q}$. For $\eta=0$ this reduces to a simple Lorentzian; we refer to this function as a Lorentzian power law (LPL).

We fit our radial data to the sum of a DS Kummer function and a LPL where the widths of the two functions, as well as their relative intensities, are determined by the $100^{\circ} \mathrm{C}$ data, with the assumption that $\eta=0$ at $100^{\circ} \mathrm{C}$. Likewise, we fit the transverse data to the sum of two LPL, where the widths and relative intensity are again determined by the low temperature lineshape. At each subsequent temperature, we simultaneously fit the radial and transverse scans with the total intensity and $\eta$ as the only adjustable parameters. These fits are shown as the solid lines in Fig. 1. Since $\eta$ is the only parameter which affects the lineshape, it is striking that these fits so accurately describe the data near the transition.

A plot of $\eta$ vs. temperature is shown as the closed circles in Fig. 2(b). We find that $\eta$ is quite small until approximately $375^{\circ} \mathrm{C}$, at which point it begins to climb, passing through 0.8 at approximately $450^{\circ} \mathrm{C}$, the same temperature at which the Bragg component of the scattering falls to zero. We note that above $T_{R}$ the power law lineshape is the theoretically expected form for the scattering (and thus $\eta$ is a measure of the decay of spatial order), whereas below $T_{R} \quad \eta$ represents only an effective parameterization of the peak-to-tail ratio. 
For $\mathrm{T} \gtrsim 500^{\circ} \mathrm{C}$, these fits become noticeably worse, presumably due to changes in $\xi_{\mathrm{x}}$ and $\xi_{\mathrm{y}}$. These lengths are infinite for $\mathrm{T}<\mathrm{T}_{\mathrm{R}}$ and decrease with temperature for $\mathrm{T}>\mathrm{T}_{\mathrm{R}}$. So long as $\xi_{\mathrm{x}}, \xi_{\mathrm{y}} \gtrsim \mathrm{L}$, the fits discussed should be valid. However, as $\xi_{\mathrm{x}}$ and $\xi_{\mathrm{y}}$ become noticeably less than $\mathrm{L}$, it becomes necessary to treat them as adjustable parameters. While this could, in principle, be accomplished [6], our signal-to-noise at high temperatures is too low to treat $\xi_{\mathrm{x}}, \xi_{\mathrm{y}}$, and $\eta$ as adjustable and obtain meaningful values for these parameters.

In the above analysis, we assume that the low temperature lineshape simply reflects a distribution of smooth domains found on the crystal surface. To test the sensitivity of our results to this assumption, we consider the possibility that the surface is exhibiting local thermal roughness at $100^{\circ} \mathrm{C}$, and that the low temperature tails are at least partially the result of atomic steps arising within a given domain (as predicted for $T \leqslant T_{R}$ ). This represents the opposite limit from the above analysis. In this case, the Bragg scattering component of the (1 $1 \overline{1} 0)$ peak is described exclusively by a Gaussian function of fixed width; the resulting Bragg intensity is shown as open triangles in Fig. 2(a). Likewise, the power law fits for the radial and transverse scans should be to a single Kummer function and a single LPL, respectively. The $\eta$ obtained from these fits are shown as open circles in Fig. 2(b). Our principal results that the Bragg scattering goes to zero and that $\eta$ remains small until near a $T_{R}$ of approximately $450^{\circ} \mathrm{C}$ remain unchanged.

It is worth noting that surface melting, as opposed to roughening, leads to a broadened Lorentzian lineshape [8]. For comparison, the data near $T_{R}$ are fit to a Lorentzian of adjustable amplitude and width. These fits are shown as dashed lines for the $450^{\circ} \mathrm{C}$ data in Fig. 1. They are noticably poorer fits; the goodness-of-fit parameter $\chi^{2}$ is approximately four times worse than that obtained for the power law lineshape. At $480^{\circ} \mathrm{C}$, where $\eta$ is close to unity, the $\chi^{2}$ of a best fit Lorenztzian is approximately two times worse than that of the power law lineshape.

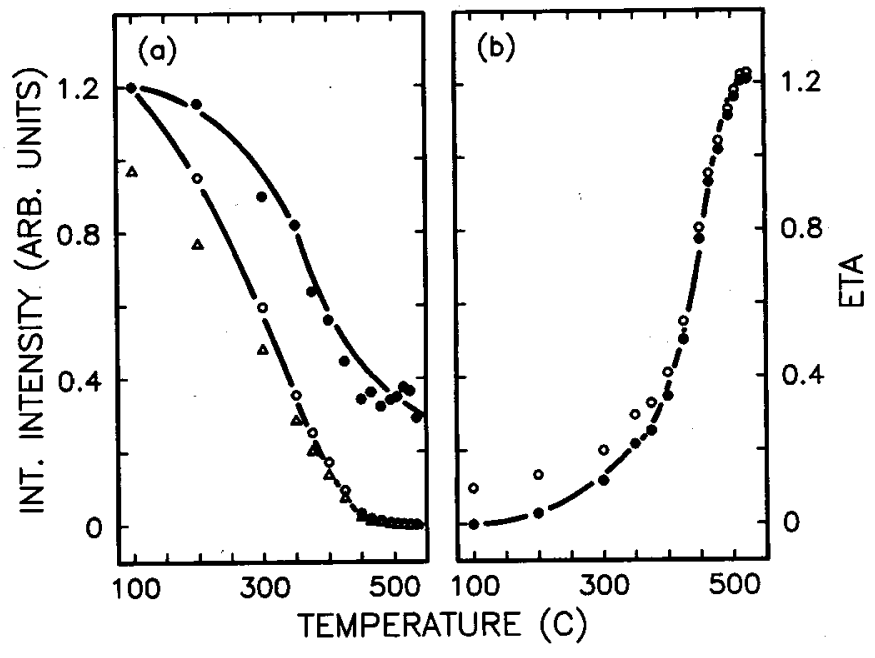

Fig. 2 - (a) Total integrated intensity of the $\mathrm{Ag}(1 \overline{1} 0)$ surface peak as a function of temperature shown as closed circles. The open circles and triangles are the integrated intensity of the Bragg ( $\delta$-function) component of the peak assuming that the low temperature $\left(100^{\circ} \mathrm{C}\right)$ surface is thermally smooth and rough, respectively (see text). (b) The exponent $\eta$ as a function of temperature as determined by best fits to power law lineshapes. The closed and open circles are the values of $\eta$ obtained assuming that the low temperature surface is thermally smooth and rough, respectively (see text). 


\section{$\underline{\operatorname{GOLD}(110)}$}

Many noble metal facets are known to favor reconstructed structures at low temperatures. Further, at some temperature $T_{c}$ a reconstructed surface will typically undergo a reversible "deconstruction" to a high temperature structure which is no longer reconstructed. One such transition which has been studied theoretically [9] as well as with electron diffraction $[10,11]$ is the $\mathrm{Au}(110) 1 \times 2$ to $1 \times 1$ deconstruction. While the "missing row" model of the reconstructed $1 \times 2$ surface is well established [10-12], the nature of the deconstruction transition itself remains controversial $[9-11]$.

For our $\mathrm{Au}(110)$ surface, representative scans along $(00 \ell)$ are shown in Fig. 3. As may be inferred from Fig. 3(a), at $400^{\circ} \mathrm{C}$ the equilibrium structure of our $\mathrm{Au}(110)$ surface was a $1 \times 3$ reconstruction. Peaks were observed at $(1 \overline{1} 0)$ and $(00 \ell / 3)$ for $\ell=1,2, \ldots, 7$, with no other peaks along the $(00 \ell)$ axis observed above the background. The $1 \times 3$ reconstruction was also observed with in situ LEED. Typically, ion sputtering at room temperature resulted in a $1 \times 2$ reconstruction. Annealing to $300^{\circ} \mathrm{C}$ resulted in an improved $1 \times 2$ surface. However, at temperatures above $300^{\circ} \mathrm{C}$, the surface evolved from a $1 \times 2$ to a $1 \times 5$ to a $1 \times 3$ reconstruction. Upon cooling, a highly ordered $1 \times 3$ reconstruction would remain with surface domains of at least $300 \AA$ and $1500 \AA$ along the (001) and $(1 \overline{1} 0)$ directions, respectively [13]. At $485^{\circ} \mathrm{C}$, the $1 \times 3$ reconstruction would undergo a reversible deconstruction; it is this transition which we have studied in depth.

It remains unclear precisely why the equilibrium reconstruction of our surface is $1 \times 3$. While observations of a $1 \times 3$ reconstruction of $\mathrm{Au}(110)$ have been reported [14], other researchers appear to find the $1 \times 2$ phase dominant $[10-12]$. We note that following our x-ray scattering experiment we remounted the gold crystal in a different UHV chamber which contained both LEED and cylindrical mirror Auger (CMA) diagnostics. Annealing the crystal for 3 hours at $300^{\circ} \mathrm{C}$ in this chamber resulted in a $1 \times 2$ LEED pattern, while annealing at $430^{\circ} \mathrm{C}$ for 12 hours (at a pressure of $1 \times 10^{-10} \mathrm{Torr}$ ) resulted in a $1 \times 3$ reconstruction. In both cases, no impurities were detected with the CMA analyzer. Following these observations, submonolayer coverages of $\mathrm{K}$ were evaporated onto the crystal surface as part of an unrelated experiment. Following that experiment, we sputtered the sample continuously for 1 week at $600^{\circ} \mathrm{C}$. After this vigorous cleaning, subsequent sputtering and annealing cycles always resulted in a $1 \times 2$ reconstruction. Thus, it appears likely that trace impurities (which diffuse to the surface of our crystal during annealing) are responsible for the observed $1 \times 3$ reconstruction. However, the fact that no impurities were detected with the CMA analyzer suggests that any such impurities are present in concentrations $\$ 0.02$ monolayer. That such low coverages could alter the equilibrium reconstruction of the $\mathrm{Au}(110)$ surface is consistent with experimental observations [15] as well as calculations by Ercolessi et al [16] which find that the $1 \times 2$ and $1 \times 3$ reconstructions should have comparable energies. Further, the large distances over which the $1 \times 3$ reconstruction is coherent indicate that any surface impurities simply stabilize this reconstruction; they do not introduce a random disorder. This crystal has therefore provided the unique opportunity to compare the statistical physics of the $1 \times 2$ and $1 \times 3$ reconstructions of Au (110) -- surfaces which are closely similar [16]. We emphasize that because $1 \times 3$ is the equilibrium structure for the experiments reported here, we are concomitantly studying the equilibrium phase transition between this reconstruction and its disordered phase.

A missing row model of the $1 \times 3$ reconstruction is shown in Fig. 4(a). If the atoms remain in their bulk positions, then the $(00 \ell / 3)$ surface diffraction peaks with $\ell=1,5,7$ should have identical intensities (excluding the usual geometric factors), the $\ell=2,4$ peaks should have zero intensity and the intensity of the $\ell=3$ peak should be 0.0625 times that of the $\ell=5$ peak. In fact, all of the $(00 \ell / 3)$ peaks are observed, and the intensities of the $\ell=1,5$, and 7 peaks vary by about an order of magnitude. If one allows $10 \%$ and $7 \%$ in-plane distortions of the 2 nd and 3 rd row atoms, respectively (as indicated in Fig. 4(a)), the resultant structure predicts a set of intensities which are in excellent agreement with the measured values of all superlattice and surface peaks. Ion channeling studies have also observed this structure (with additional distortions normal to the surface, which do not affect our in-plane intensities) for a $1 \times 3$ reconstruction induced on $\mathrm{Au}(110)$ by a 0.05 monolayer coverage of Cs [15]. We assume this to be the structure of the $1 \times 3$ reconstruction, although our conclusions concerning the nature of the deconstruction transition do not depend on this assumption. 


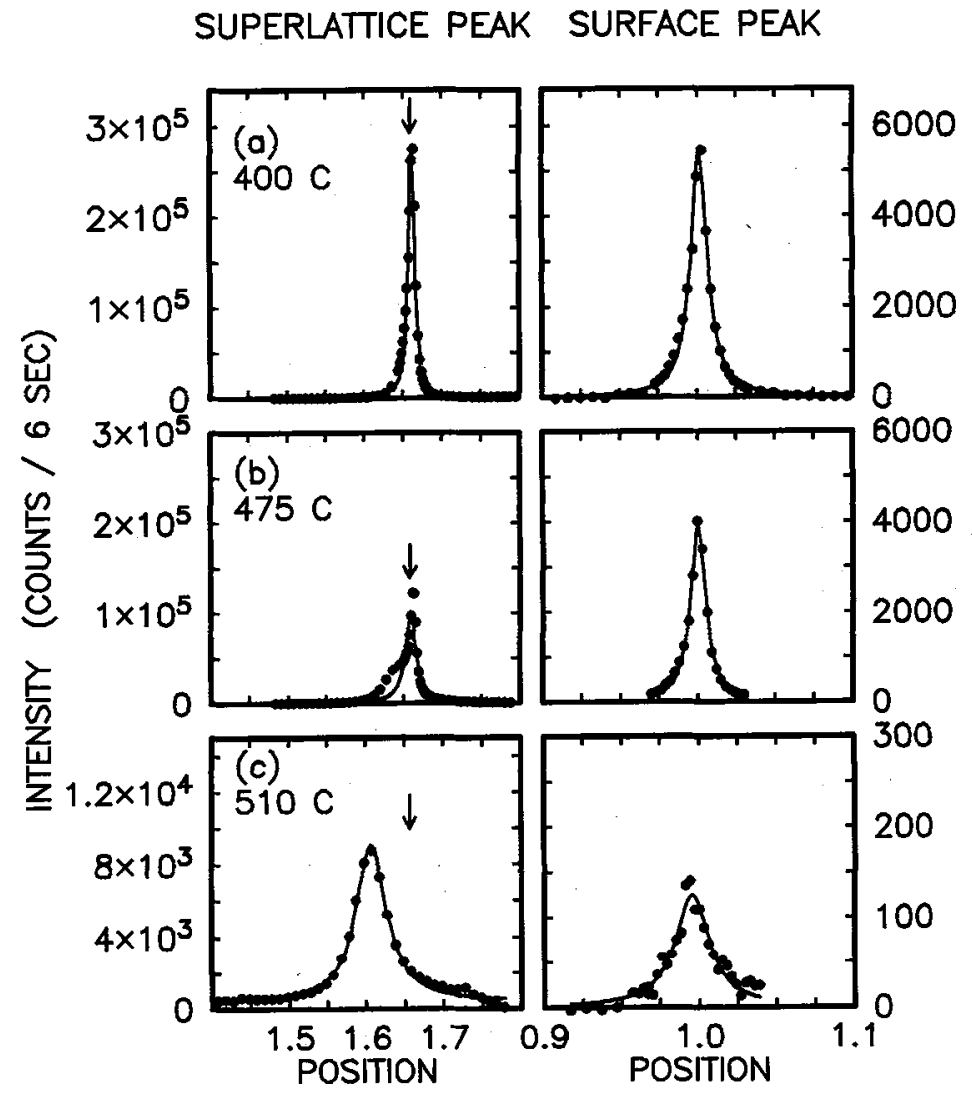

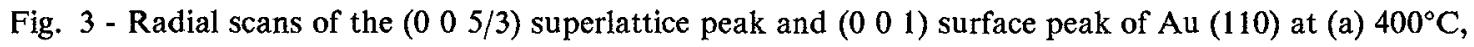
(b) $475^{\circ} \mathrm{C}$, and (c) $510^{\circ} \mathrm{C}$; below, near, and above the commensurate-incommensurate transition, respectively. The lineshapes are best fit Lorentzians, with a constant background subtracted from all of the scans and thermal diffuse scattering peaked at $\left(\begin{array}{lll}0 & 0 & 2\end{array}\right)$ subtracted from the $\left(\begin{array}{lll}0 & 0 & 5 / 3\end{array}\right)$ data. A second peak in the low wavevector shoulder of the $475^{\circ} \mathrm{C}$ superlattice data is observable (but not fitted) and is discussed in the text. The units of peak position are $2 \pi / a_{0}=1.539 \AA^{-1}$. The arrows indicate the $(005 / 3)$ positions commensurate with the bulk lattice.

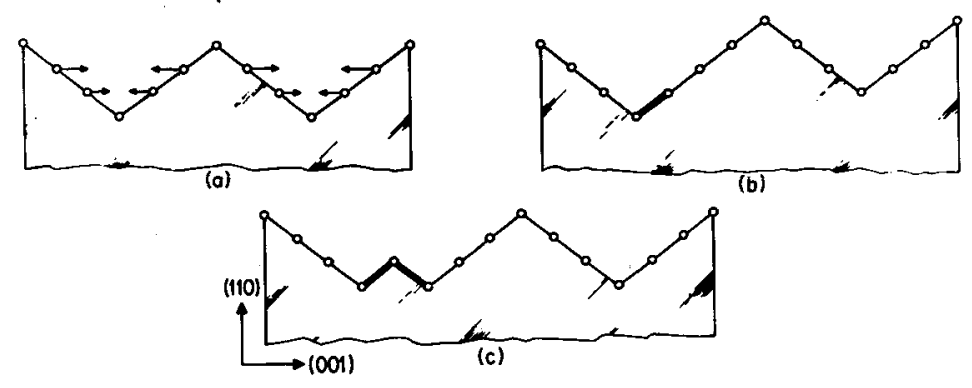

Fig. 4 - (a) Schematic representation of the Au(110) $1 \times 3$ missing-row reconstruction. The arrows indicate the outward atomic distortions observed in the second and third rows from the surface. (b) A single atomic step thermal excitation of the $1 \times 3$ surface. (c) A paired-step thermal excitation of the $1 \times 3$ surface. 
As is evident in Fig. 3(b) and (c), the superlattice peak positions shift between $475^{\circ} \mathrm{C}$ and $510^{\circ} \mathrm{C}$. In Fig. 5, we plot the position and width of the $(005 / 3)$ peak as a function of temperature. The position remains essentially constant until $485^{\circ} \mathrm{C}$, at which point the superlattice becomes incommensurate with the bulk. As the $(005 / 3)$ peak becomes increasingly incommensurate, it also becomes increasingly broad (Fig. 5), indicating a decrease in the size of the spatially coherent domains. This behavior can result from the proliferation of atomic steps (such as those shown in Fig. 4 (b) and (c)) which act as domain walls between coherent regions of the reconstructed surface. As the density of these domain walls increases, the surface follows a commensurate-incommensurate transition (CIT) [17]. Throughout this CIT, the $\left(\begin{array}{lll}0 & 0 & 2 / 3\end{array}\right)$ and $\left(\begin{array}{lll}0 & 0 & 5 / 3\end{array}\right)$ peaks are shifted downward in reciprocal space by equal amounts, whereas the $\left(\begin{array}{ll}0 & 0 \\ 1 / 3\end{array}\right),\left(\begin{array}{lll}0 & 0 & 4 / 3\end{array}\right)$ and and $\left(\begin{array}{lll}0 & 0 & 7 / 3\end{array}\right)$ peaks are displaced by the same magnitude but in the opposite direction. This is consistent with the proliferation of domain walls between commensurate regions of $1 \times 3$ surface $[12,17]$.

As shown in Fig. 5, between $100^{\circ}$ and $450^{\circ} \mathrm{C}$ the superlattice peak position and width remain essentially constant, while the peak intensity decreases by $15 \%$. Empirically, the $400^{\circ}$ and $510^{\circ} \mathrm{C}$ peaks are well described by a Lorentzian lineshape (Fig. 3(a) and (c)). However, the $475^{\circ} \mathrm{C}$ data clearly have a second peak in the low wavevector shoulder of the best fit Lorentzian shown in Fig. 3(b). The appearance of a second peak at temperatures near $T_{c}$ could result from a distribution of transition temperatures (as has been shown for other CIT's [18] ), long equilibration times or, possibly, a two-phase coexistence.

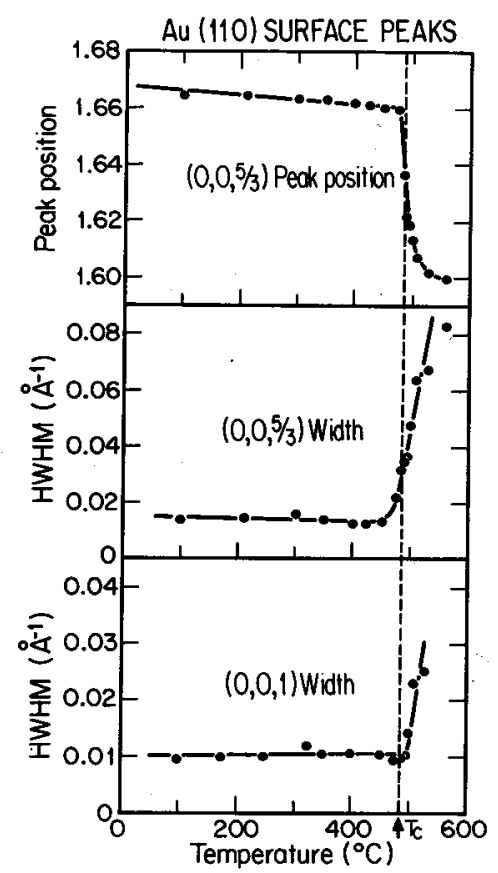

Fig. 5 - Position and width of the $\left(\begin{array}{lll}0 & 0 & 5 / 3\end{array}\right)$ superlattice peak and width of the $\left(\begin{array}{lll}0 & 0 & 1\end{array}\right)$ peak of $\mathrm{Au}\left(\begin{array}{ll}110\end{array}\right)$ as a function of temperature. These are deduced from best fits to a single Lorentzian. The units of peak position are $2 \pi / a_{o}=1.539 \AA^{-1}$. The gradual decrease in peak position between 100 and $475^{\circ} \mathrm{C}$ is due to thermal expansion of the crystal lattice. Between $450^{\circ}$ and $475^{\circ} \mathrm{C}$, a second peak in the shoulder of the $(005 / 3)$ data (see Fig. 3(b) and text) results in an apparent broadening of the best fit Lorentzian. 
While the shift and broadening of the superlattice peaks indicate the formation of atomic steps between domains of the reconstructed surface, this information does not enable us to distinguish between single atomic steps and pairs of atomic steps, such as those shown in Figs. 4(b) and (c), respectively. Villain and Vilfan [9] have shown for the $\mathrm{Au}(110) 1 \times 2$ reconstructed surface that paired steps could deconstruct the surface without roughening it, while the proliferation of single atomic steps would both roughen and deconstruct the surface. The same is true of the $1 \times 3$ surface.

To distinguish between single and paired atomic steps, it is necessary to consider the behavior of the integral order (001) surface peak. The domains on both sides of the paired step excitation in Fig. 4(c) are in phase with respect to this peak, whereas those separated by the single step excitation of Fig. 4(b) are precisely out of phase. If only paired steps were present, one would observe broadening of the fractional superlattice peaks, but not the integral ones. However, we find that above the CIT both the (001) and (110) surface peaks show significant broadening in both the radial and transverse directions, as well as a loss of peak intensity comparable to that of the superlattice peaks. This is shown quantitatively in Fig. 5. The broadening of the $\left(\begin{array}{lll}0 & 0 & 1\end{array}\right)$ peak demonstrates that single step excitations are indeed present, the apparent width of this peak being inversely proportional to the distance between unpaired steps. The greater broadening of the $(005 / 3)$ peak suggests that some paired step excitations are present as well. Further, the broadening in both the $\left(\begin{array}{lll}0 & 0 & 1\end{array}\right)$ and $(1 \overline{1} 0)$ directions indicates that the excitations are meandering along the $\left(\begin{array}{lll}0 & 0 & 1\end{array}\right)$ direction, as expected for a roughened surface [9].

The observed evolution of the surface peaks is consistent with a surface roughening transition. As with $\mathrm{Ag}(110)$, the scattering from a smooth surface should exhibit $\delta$-function lineshapes; as the surface roughened, these $\delta$-functions would evolve into power law lineshapes [3]. Between the smooth and rough states, only the power law exponent should change; the linewidth (which results from resolution and finite size effects) should remain constant. However, in practice, most power law lineshapes with exponent $0.25 \$ \eta \$ 0.5$ can be well parameterized by a Lorentzian [8]. As the exponent of the power law increases, so does the effective width of the best Lorentzian fit.

We find that at all temperatures, our radial and transverse scans are well described by Lorentzian lineshapes (except just below $\mathrm{T}_{\mathfrak{c}}$, where the radial superlattice data are double peaked, as discussed above). At low temperatures, the Lorentzian tails may be the result of either a surface miscut [19] or the surface mosaic, which in our resolution limit is convolved into both the radial and transverse scans. At higher temperatures, when the tails become more pronounced, we believe that we are in fact observing a change in lineshape. Quite possibly, these tails are due to the power law lineshape expected for a roughened surface. While we do not attach any physical significance to the observed Lorentzian lineshapes, these fits do provide a systematic means of parameterizing the evolution of peak position, width, and intensity.

The universality class of the deconstruction transition is closely related to the type of excitation which causes the deconstruction [20]. For the $1 \times 3 \mathrm{Au}(110)$ surface, a deconstruction via paired steps would be of the 3-state chiral Potts universality class. However, the proliferation of uncorrelated single step excitations should theoretically result in logarithmically divergent height fluctuations associated with a roughening transition. The resulting simultaneous deconstruction and roughening would be of the Kosterlitz-Thouless universality class, independent of the presence or absence of paired step excitations. Our data are only consistent with the second scenario. However, further studies will be needed to quantify the relevant critical properties and thus confirm the universality class of this transition. 


\section{CONCLUSIONS AND ACKNOWLEDGEMENTS}

In conclusion, we have found direct, model-independent evidence for a transition between smooth and logarithmically rough phases of a clean $\mathrm{Ag}(110)$ metal surface. For the $\mathrm{Au}(110)$ surface, we have found that the reversible deconstruction of the $1 \times 3$ reconstruction is the result of the concurrent proliferation of single and paired atomic-step excitations, suggesting that the roughening and deconstruction of this surface are, in fact, a single process. Further, our results demonstrate that glancing angle $\mathrm{x}$-ray scattering can provide direct information about thermal variations of the surface height-height correlation function.

We wish to thank W. Haag for technical assistance and and T. Gustafsson for supplying the Au crystal used in this work. Part of the work was performed at SSRL which is supported by the Department of Energy, Office of Basic Energy Sciences. The MIT component of this research was supported by the Joint Services Electronics Program under Contract No. DAAL-86-K0002.

\section{REFERENCES}

1. S. G. J. Mochrie, Phys. Rev. Lett. 59, 304 (1987); G. A. Held, et al, ibid 59, 2075 (1987); K. S. Liang, et al, ibid 59, 2447 (1987).

2. For example, J. D. Weeks in Ordering in Strongly Fluctuating Condensed Matter Systems, edited by T. Riste (Plenum, New York, 1980), p. 293.

3. J. Villain, D. R. Grempel and J. Lapujoulade, J. Phys. F. 15, 809 (1985); M. den Nijs, et. al., Phys. Rev. Lett. 55, 1689 (1985); 57, 1279 (1986) (erratum); A. Trayanov, A. C. Levi, and E. Tosatti, submitted for publication.

4. Precisely, the (1T⿱100) surface peak is the anti-Bragg point along the $(\ell+1 \quad \ell-1 \quad 0)$ crystal truncation rod. To obtain a non-zero incident flux (and thus a non-zero scattering intensity), it was necessary to study the $(1.03 \overline{0.97} 0)$ point on this truncation rod, which, for simplicity, we refer to as the (1 $\overline{1} 0)$ surface peak. The radial and transverse scans of Fig. 1 are thus of the form $(1.03+\delta, \overline{0.97+\delta, 0)}$ and $(1.03, \overline{0.97}, \delta)$, respectively. Likewise, the (001) surface peak is the anti-Bragg point along the $(\ell \ell 1)$ crystal truncation rod and the radial scans of Fig. 3 are of the form $\left(\begin{array}{lll}0.03 & 0.03 & 1+\delta\end{array}\right)$ and $\left(\begin{array}{lll}0.03 & 0.03 & 5 / 3\end{array}+\delta\right)$, for the integral and superlattice peaks, respectively. See, I. K. Robinson, Phys. Rev. B 33, 3038 (1986); S. G. Andrews and R. A. Cowley, J. Phys. C 186427 (1985).

5. G. A. Held, J. L. Jordan-Sweet, P. M. Horn, A. Mak, and R. J. Birgeneau, accepted for publication, Solid State Comm.

6. S. G. J. Mochrie, A. R. Kortan, R. J. Birgeneau, and P. M. Horn, Z. Phys. B 62, 79 (1985).

7. P. Dutta and S. K. Sinha, Phys. Rev. Lett. 47, 50 (1981).

8. P. A. Heiney et al, Phys. Rev. B 28, 6416 (1983).

9. J. Villain and I. Vilfan, Surf. Sci. 199, 165 (1988).

10. J. C. Campuzano, M. S. Foster, G. Jennings, R. F. Willis and W. Unertl, Phys. Rev. Lett. 54, 2684 (1985).

11. D. Wolf, H. Jagodzinski and W. Moritz, Surf. Sci. 77, 265 (1978); 77, 283 (1978); E. G. McRae, T. M. Buck, R. A. Malic, and G. H. Wheatley, Phys. Rev. B 36, 2341 (1987); W. Moritz and D. Wolf, Surf. Sci. 163, L655 (1985); J. Noonan and H. L. Davis, J. Vac. Sci. Technol. 16, 587 (1979).

12. I. K. Robinson, Phys. Rev. Lett. 50, 1145 (1983); I. K. Robinson, Y. Kuk and L. C. Feldman, Phys. Rev. B 29, 4762 (1984).

13. These domain sizes are determined from the half-widths of the radial and transverse (001) surface peaks, respectively, which due to resolution effects only set a lower limit on these coherence lengths. The observed surface mosaic was $0.06^{\circ} \mathrm{HWHM}$.

14. W. Moritz and D. Wolf, Surf. Sci. 88, L29 (1979); G. Bennig et al, Surf. Sci. 131, L379 (1983).

15. P. Häberle, P. Fenter, and T. Gustafsson, Phys. Rev. B 39, 5810 (1989). 
16. F. Ercolessi, et al, Surf. Sci. 189/190, 636 (1987).

17. For a review, see P. Bak, Rep. Prog. Phys. 45, 587 (1982); see also, A. Erbil et al, Phys. Rev. B 28, 6329 (1983).

18. P. W. Stephens et al, Phys. Rev. B 29, 3512 (1984).

19. M. Presicci and T. M. Lu, Surf. Sci. 141, 233 (1984).

20. P. Bak, Physica 99B, 325 (1980). 